\title{
Charge current in ferromagnet-superconductor junction with pairing state of broken time-reversal symmetry.
}

\author{
N. Stefanakis \\ Department of Physics, University of Crete, P.O. Box 2208, GR-71003, Heraklion, Crete, Greece
}

(October 30, 2018)

\begin{abstract}
We calculate the tunneling conductance spectra of a ferromagnetic metal/insulator/superconductor using the Blonder-Tinkham-Klapwijk (BTK) formulation. Two possible states for the superconductor are considered with the time reversal symmetry $(\mathcal{T})$ broken, i.e., $d_{x^{2}-y^{2}}+i s$, or $d_{x^{2}-y^{2}}+i d_{x y}$. In both cases the tunneling conductance within the gap is suppressed with the increase of the exchange interaction due to the suppression of the Andreev reflection. In the $\left(d_{x^{2}-y^{2}}+i s\right)$-wave case the peaks that exist when the ferromagnet is a normal metal in the amplitude of the $s$-wave component due to the bound state formation are reduced symmetrically, with the increase of the exchange field, while in the $\left(d_{x^{2}-y^{2}}+i d_{x y}\right)$-wave case the residual density of states within the gap develops a dip around $E=0$ with the increase of the exchange field. These results would be useful to discriminate between $\mathcal{T}$-broken pairing states near the surface in high- $T_{c}$ superconductors.

74.20. z, 74.50.+r, 74.80.Fp
\end{abstract}

Typeset using REVTEX 


\section{INTRODUCTION}

It is generally accepted that the pairing state of high- $T_{c}$ cuprate superconductors is of dominant $\left(d_{x^{2}-y^{2}}\right)$-wave symmetry. However, it is possible for a secondary component to be induced wherever the dominant order parameter varies spatially, for example, in regions close to surfaces [1]. The local pairing symmetry can be detected by tunneling measurements where Andreev reflection take place [2, 3]. In the Andreev reflection process an electron incident, in the barrier with an energy below the superconducting gap can not drain off into the superconductor. It is instead reflected as a hole and a Cooper pair is transferred into the superconductor. In anisotropic high- $T_{c}$ superconductors the transmitted quasiparticles experience different sign of the pair potential. This results in the formation of bound states close to the surface [4], which are detected as zero energy peaks in the tunneling spectra as an indication of $\left(d_{x^{2}-y^{2}}\right)$-wave pairing symmetry [5]. The splitting of the zero energy conductance peak at low temperatures observed experimentally [6.7] is a signature of a two component pairing state with the time reversal symmetry broken, and is consistent with both the $d_{x^{2}-y^{2}}+i s$, or $d_{x^{2}-y^{2}}+i d_{x y}$ pairing state. The tunneling spectra of a normal metal/insulator/superconductor junctions, for the above pairing states has already been calculated, by extending the Blonder-Tinkham-Klapwijk (BTK) theory to include the anisotropy of the pair potential [8]. Based on the bound state formation, simple arguments have been derived to explain the subgap conductance in each case and to discriminate between the two pairing symmetries.

Moreover the tunneling spectra of ferromagnet/superconductor junctions has been clarified both for singlet [9 12], and triplet [13] pairing states where the important parameter is the exchange field. Also the changes of the critical temperature of the superconductor in $s$-wave-superconductor- ferromagnet multilayers have been studied [14]. In this paper we use the scattering approach to study the charge transport through ferromagnet/superconductor with time reversal $\mathcal{T}$-breaking pairing states junctions. For the superconductor we assume two pairing states i.e. $d_{x^{2}-y^{2}}+i s$, or $d_{x^{2}-y^{2}}+i d_{x y}$, with a complex order parameter breaking 
the time reversal symmetry. In both cases the tunneling conductance within the gap is suppressed with the increase of the exchange interaction, due to the suppression of the Andreev reflection. In the $\left(d_{x^{2}-y^{2}}+i s\right)$-wave case the peaks that exist when the ferromagnet is a normal metal in the amplitude of the $s$-wave component due to the bound state formation are reduced symmetrically. In the $\left(d_{x^{2}-y^{2}}+i d_{x y}\right)$-wave case the tunneling conductance within the gap develops a dip around $E=0$ with the increase of the exchange field. The tunneling conductance characteristics for $\mathcal{T}$-broken pairing states are discussed extensively in Sec. III.

The evolution of the Andreev- and normal-reflection amplitude with the exchange field presented in Sec. IV explains the suppression of the tunneling conductance with the exchange field. The Andreev reflection amplitude decays to zero at a critical value of the exchange

field $x_{c}$ that depends only on the angle $\theta$ of the quasiparticle trajectory. A bound state at $\theta$ contributes to the Andreev reflection and to the conductance for a given $x$ only when $x<x_{c}$.

The magnetic field for finite exchange interaction induces an imbalance in the peak heights, of the spectra for energy positive and negative as seen in Sec. V.

Also the $c$-axis tunneling spectra presented in the last section is similar for $\mathcal{T}$ broken pairing states, since the pairing potential along the $c$-axis does not change sign on the Fermi surface. These results would be helpful to discriminate between time-reversal symmetry broken surface states in high $T_{c}$ superconductors.

\section{THEORY OF TUNNELING EFFECT}

The motion of quasiparticles in inhomogeneous superconductors is described by the Bogoliubov de Gennes (BdG) equations. The motion of electrons inside the ferromagnet is described within the Stoner model by an effective single particle Hamiltonian with an exchange interaction. The BdG equations read [15]

$$
\begin{aligned}
& \left(\mathcal{H}_{e}(\boldsymbol{r})-\rho U(\boldsymbol{x})\right) u(\boldsymbol{r})+\int d \boldsymbol{r}^{\prime} \Delta(\boldsymbol{s}, \boldsymbol{x}) v\left(\boldsymbol{r}^{\prime}\right)=E u(\boldsymbol{r}) \\
& \int d \boldsymbol{r}^{\prime} \Delta^{*}(\boldsymbol{s}, \boldsymbol{x}) u\left(\boldsymbol{r}^{\prime}\right)-\left(\mathcal{H}_{e}^{*}(\boldsymbol{r})+\rho U(\boldsymbol{x})\right) v(\boldsymbol{r})=E v(\boldsymbol{r})
\end{aligned}
$$


The single-particle Hamiltonian is given by $\mathcal{H}_{e}(\boldsymbol{r})=-\hbar^{2} \nabla_{\boldsymbol{r}}^{2} / 2 m_{e}+V(\boldsymbol{r})-E_{F}, E$ is the energy measured from the Fermi energy $E_{F} \cdot U(\boldsymbol{r})$ is the exchange potential, $\rho$ is $1(-1)$ for $\operatorname{up}($ down $)$-spins. $\Delta(\boldsymbol{s}, \boldsymbol{x})$ is the pair potential, after a transformation from the position coordinates $\boldsymbol{r}, \boldsymbol{r}^{\prime}$ to the center of mass coordinate $\boldsymbol{x}=\left(\boldsymbol{r}+\boldsymbol{r}^{\prime}\right) / 2$ and the relative vector $\boldsymbol{s}=\boldsymbol{r}-\boldsymbol{r}^{\prime}$. After Fourier transformation the pair potential depends on the related wave vector $\boldsymbol{k}$ and $\boldsymbol{x}$. In the weak coupling limit $\boldsymbol{k}$ is fixed on the Fermi surface $\left(|\boldsymbol{k}|=k_{F}\right)$, and only its direction $\theta$ is variable. After applying the quasi-classical approximation, i.e., [15]

$$
\left(\begin{array}{l}
\bar{u}(\boldsymbol{r}) \\
\bar{v}(\boldsymbol{r})
\end{array}\right)=e^{-i \boldsymbol{k} \cdot \boldsymbol{r}}\left(\begin{array}{c}
u(\boldsymbol{r}) \\
v(\boldsymbol{r})
\end{array}\right),
$$

so that the fast oscillating part, of the wave function is divided out, the BdG equations are reduced to the Andreev equations [3]

$$
\begin{aligned}
& E \bar{u}(\boldsymbol{r})=-i v_{F} \boldsymbol{k} \cdot \nabla \bar{u}(\boldsymbol{r})+\Delta(\theta, \boldsymbol{r}) \bar{v}(\boldsymbol{r}) \\
& E \bar{v}(\boldsymbol{r})=i v_{F} \boldsymbol{k} \cdot \nabla \bar{v}(\boldsymbol{r})+\Delta^{*}(\theta, \boldsymbol{r}) \bar{u}(\boldsymbol{r})
\end{aligned},
$$

where the quantities $\bar{u}(\boldsymbol{r})$ and $\bar{v}(\boldsymbol{r})$ are electronlike and holelike quasiparticles and $v_{F}$ is the Fermi velocity.

We consider the ferromagnet/insulator/superconductor junction shown in Fig. 1. We choose the $y$ direction to be parallel to the interface, and the $x$ direction to be normal to the interface. The insulator is modeled by a $\delta$ function, located at $x=0$, of the form $V \delta(x)$. The temperature is fixed to $0 \mathrm{~K}$. We take both the pair potential and the exchange energy as a step function i.e. $\Delta(\theta, \boldsymbol{r})=\Theta(x) \Delta(\theta), U(\boldsymbol{r})=\Theta(-x) U$. For the geometry shown in Fig. 1, Eqs. 3 take the form

$$
\begin{aligned}
& E \bar{u}(x)=-i v_{F} k_{F x} \frac{d}{d x} \bar{u}(x)+\Delta(\theta) \bar{v}(x) \\
& E \bar{v}(x)=i v_{F} k_{F x} \frac{d}{d x} \bar{v}(x)+\Delta^{*}(\theta) \bar{u}(x)
\end{aligned}
$$

When a beam of electrons is incident from the ferromagnet to the insulator, with an angle $\theta$, the general solution of Eqs. (4), is the two component wave function $\Psi_{I}$ that for $x<0$ is written as 


$$
\Psi_{I}=\left(\begin{array}{l}
1 \\
0
\end{array}\right) e^{i q_{\uparrow[\downarrow]} x \cos \theta}+a_{\uparrow[\downarrow]}\left(\begin{array}{l}
0 \\
1
\end{array}\right) e^{i q_{\downarrow[\uparrow]} x \cos \theta_{A}}+b_{\uparrow[\downarrow]}\left(\begin{array}{l}
1 \\
0
\end{array}\right) e^{-i q_{\uparrow[\downarrow]} x \cos \theta},
$$

where $a_{\uparrow[\downarrow]}, b_{\uparrow[\downarrow]}$, are the amplitudes for Andreev and normal reflection for spin-up(-down) quasiparticles, and $q_{\uparrow[\downarrow]}=\sqrt{\frac{2 m}{\hbar^{2}}\left(E_{F} \pm U\right)}$ is the wave vector of quasiparticles in the ferromagnet for up (down-)-spin. The wave vector of the electronlike, holelike quasiparticles is approximated by $k_{s}=\sqrt{\frac{2 m E_{F}}{\hbar^{2}}}$. Since the translational symmetry holds in the $y$-axis direction, the momenta parallel to the interface is conserved, i.e., $q_{\uparrow} \sin \theta=q_{\downarrow} \sin \theta_{A}=k_{s} \sin \theta_{s}$. Note that $\theta$ is different than $\theta_{A}$ since the retroreflection of the Andreev reflection is broken. Using the matching conditions of the wave function at $x=0, \Psi_{I}(0)=\Psi_{I I}(0)$ and $\Psi_{I I}^{\prime}(0)-\Psi_{I}^{\prime}(0)=\left(2 m V / \hbar^{2}\right) \Psi_{I}(0)$, the Andreev- and normal-reflection amplitudes $a_{\uparrow[\downarrow]}, b_{\uparrow[\downarrow]}$ for the spin-up(-down) quasiparticles are obtained as

$$
\begin{aligned}
& a_{\uparrow[\downarrow]}=\frac{4 n_{+} \lambda_{1}}{\left(-1-\lambda_{1}-i z_{\uparrow[\downarrow]}\right)\left(-1-\lambda_{2}+i z_{\uparrow[\downarrow]}\right)+\left(1-\lambda_{1}-i z_{\uparrow[\downarrow]}\right)\left(-1+\lambda_{2}-i z_{\uparrow[\downarrow]}\right) n_{+} n_{-} \phi_{-} \phi_{+}^{*}}, \\
& b_{\uparrow[\downarrow]}=\frac{\left(-1-\lambda_{2}+i z_{\uparrow[\downarrow]}\right)\left(1-\lambda_{1}+i z_{\uparrow[\downarrow]}\right)+\left(-1+\lambda_{2}-i z_{\uparrow[\downarrow]}\right)\left(-1-\lambda_{1}+i z_{\uparrow[\downarrow]}\right) n_{+} n_{-} \phi_{-} \phi_{+}^{*}}{\left(-1-\lambda_{1}-i z_{\uparrow[\downarrow]}\right)\left(-1-\lambda_{2}+i z_{\uparrow[\downarrow]}\right)+\left(1-\lambda_{1}-i z_{\uparrow[\downarrow]}\right)\left(-1+\lambda_{2}-i z_{\uparrow[\downarrow]}\right) n_{+} n_{-} \phi_{-} \phi_{+}^{*}},
\end{aligned}
$$

where $z_{0}=\frac{m V}{\hbar^{2} k_{s}}, z_{\uparrow[\downarrow]}=\frac{2 z_{0}}{\cos \theta_{s}}, \lambda_{1}=\frac{\cos \theta}{\cos \theta_{s}} \frac{q_{\uparrow[\downarrow]}}{k_{s}}, \lambda_{2}=\frac{\cos \theta_{A}}{\cos \theta_{s}} \frac{q_{\downarrow \downarrow \uparrow]}}{k_{s}}$. The BCS coherence factors are given by

$$
\begin{aligned}
& u_{ \pm}^{2}=\left[1+\sqrt{E^{2}-\left|\Delta_{ \pm}(\theta)\right|^{2}} / E\right] / 2, \\
& v_{ \pm}^{2}=\left[1-\sqrt{E^{2}-\left|\Delta_{ \pm}(\theta)\right|^{2}} / E\right] / 2,
\end{aligned}
$$

and $n_{ \pm}=v_{ \pm} / u_{ \pm}$. The internal phase coming from the energy gap is given by $\phi_{ \pm}=$ $\left[\Delta_{ \pm}(\theta) /\left|\Delta_{ \pm}(\theta)\right|\right]$, where $\Delta_{+}(\theta)=\Delta(\theta)\left(\Delta_{-}(\theta)=\Delta(\pi-\theta)\right)$, is the pair potential experienced by the transmitted electronlike (holelike) quasiparticle.

When $\theta>\sin ^{-1}\left(\frac{k_{s}}{q_{\uparrow}}\right) \equiv \theta_{c 1}$ total reflection occurs and the spin and charge current vanishes. In the space of $\theta, x$ in Fig. 2, the dotted line from the solution of the equation $\theta=\sin ^{-1} \frac{1}{\sqrt{1+x}}$, 
where $x=U / E_{F}$, defines the boundary of the region (labeled as TR) where total reflection occurs. When $\theta_{c 1}>\theta>\sin ^{-1}\left(\frac{q_{\uparrow}}{q_{\downarrow}}\right) \equiv \theta_{c 2}$ although the transmitted quasiparticles in the superconductor, do propagate, the Andreev reflected quasiparticles, do not propagate. This process is called virtual Andreev reflection (VAR) process [10]. In this case the spin and charge current do not vanish since a finite amplitude of the Andreev reflection still exists. For $\theta<\theta_{c 2}$ Andreev reflection occurs. In Fig. 2 the solid line determined by the equation in the above inequality, i.e., $\theta=\sin ^{-1} \sqrt{\frac{1+x}{1-x}}$, separates the region where the VAR process occurs (labeled as VAR) from the region where Andreev reflection occurs (labeled as AR). A symmetric branch that is not presented in the figure occurs for negative angles $\theta$.

According to the BTK formula the conductance for the charge current of the junction, $\bar{\sigma}_{q_{\uparrow[\downarrow]}}(E, \theta)$, for up (down-)-spin quasiparticles, is expressed in terms of the probability amplitudes $a_{\uparrow[\downarrow]}, b_{\uparrow[\downarrow]}$ as [2, 10]

$$
\bar{\sigma}_{q_{\uparrow[\downarrow]}}(E, \theta)=R e\left[1+\frac{\lambda_{2}}{\lambda_{1}}\left|a_{\uparrow[\downarrow]}\right|^{2}-\left|b_{\uparrow[\downarrow]}\right|^{2}\right] .
$$

The tunneling conductance, normalized by that in the normal state is given by

$$
\begin{gathered}
\sigma_{q}(E)=\sigma_{q_{\uparrow}}(E)+\sigma_{q_{\downarrow}}(E) \\
\sigma_{q_{\uparrow \downarrow \downarrow]}}(E)=\frac{1}{R_{N}} \int_{-\pi / 2}^{\pi / 2} d \theta \cos \theta \bar{\sigma}_{q_{\uparrow\lceil\downarrow]}}(E, \theta) P_{\uparrow[\downarrow]} q_{\uparrow[\downarrow]},
\end{gathered}
$$

where

$$
\begin{gathered}
R_{N}=\int_{-\pi / 2}^{\pi / 2} d \theta \cos \theta\left[\sigma_{N_{\uparrow}}(\theta) P_{\uparrow} q_{\uparrow}+\sigma_{N_{\downarrow}}(\theta) P_{\downarrow} q_{\downarrow}\right], \\
\sigma_{N_{\uparrow \downarrow \downarrow]}}(\theta)=\frac{4 \lambda_{1}}{\left(1+\lambda_{1}\right)^{2}+z_{\uparrow\lceil\downarrow]}^{2}}
\end{gathered}
$$

where $P_{\uparrow[\downarrow]}=\left(E_{F} \pm U\right) / 2 E_{F}$ is the polarization for up(down- $)$-spin. In the $z_{0}=0$ limit the interface is regarded as a weak link, showing metallic behavior while for large $z_{0}$ values the interface becomes insulating.

We consider the following cases 
a) In the $\left(d_{x^{2}-y^{2}}+i s\right)$-wave case

$$
\Delta(\theta)=\Delta_{1} \cos [2(\theta-\beta)]+i \Delta_{2}
$$

where $\beta$ is the angle between the normal to the interface and the $x$ axis of the crystal.

b) In the $\left(d_{x^{2}-y^{2}}+i d_{x y}\right)$-wave case

$$
\Delta(\theta)=\Delta_{1} \cos [2(\theta-\beta)]+i \Delta_{2} \sin [2(\theta-\beta)]
$$

where the angular form of the secondary component is obtained by the substitution of $\beta$ in the $\left(d_{x^{2}-y^{2}}\right)$-wave order parameter by $\beta+\pi / 4$.

\section{TUNNELING CONDUCTANCE CHARACTERISTICS}

In Figs. 3 and 4 we plot the tunneling conductance $\sigma_{q}(E)$ for different values of the exchange interaction $x=U / E_{F}$ (a) $z_{0}=0, \beta=0$, (b) $z_{0}=2.5, \beta=0$, and (c) $z_{0}=2.5$, $\beta=\pi / 4$. The pairing symmetry of the superconductor is $d_{x^{2}-y^{2}}+i s$ with $\Delta_{1}=\Delta_{0}$ and $\Delta_{2}=0.3 \Delta_{0}$ in Fig. 3, $d_{x^{2}-y^{2}}+i d_{x y}$ with $\Delta_{1}=\Delta_{0}$ and $\Delta_{2}=0.3 \Delta_{0}$ in Fig. 屯. For $z_{0}=0$, the subgap conductance is suppressed, with the increase of $x$, as in the case of a $d_{x^{2}-y^{2}}$-wave superconductor [10].

In the $\left(d_{x^{2}-y^{2}}+i s\right)$-wave case when the ferromagnet is normal metal (i.e., $\left.x=0\right)$, the boundary orientation is $\beta \neq 0$, and the barrier strength $z_{0}$ is large, a peak exists in the tunneling spectra in the amplitude of the secondary component due to the bound state formation. The peak height is maximum for $\beta=\pi / 4$ since the bound state is formed for all angles $\theta$ and collapses to zero for $\beta=0$. For the $d_{x^{2}-y^{2}}+i d_{x y}$ pairing state, for $x=0$ the tunneling conductance has residual values due to the formation of bound states. The bound state energies depend on the boundary orientation $\beta$ as well as on the quasiparicle angle $\theta$. The reduced height of the subgap conductance in the $\left(d_{x^{2}-y^{2}}+i d_{x y}\right)$-wave case is explained from the discrete values of the angle $\theta$ over which the bound state occurs as compared to

the range of $\theta$ values in the $\left(d_{x^{2}-y^{2}}+i s\right)$-wave case [8]. Also an enhancement appears in the 
$\left(d_{x^{2}-y^{2}}+i d_{x y}\right)$-wave state at $x=0, E=\Delta_{d_{x y}}$ for $\beta=\pi / 4$ due to the larger contribution to the spectra of the bound state at $\theta=0\left(E=\Delta_{d_{x y}}\right)$. The same peak at $\Delta_{d_{x y}}$ becomes more pronounced in a calculation including the self consistency [16].

In the Andreev-reflection process the incident electron and the Andreev reflected hole have wave vectors with opposite spins. In a normal metal the spin-up and spin-down wave vectors are equal and no spin effects occur in the Andreev reflection. However, in a ferromagnet the wave vectors for spin-up and spin-down are different and this affects the Andreev reflection. In that case the Andreev reflected hole decays exponentially for large distance in the ferromagnet and there is no interference effect between electron and hole waves. Moreover no pairs are transferred into the superconductor, and there is weak or no interference between the transmitted quasiparticles in the superconductor. In this sense the ferromagnet does not allow the quasiparticles to enter into the superconductor, and to experience the sign change of the pair potential, which is the main reason for the tunneling peaks. As a consequence the conductance peaks disappear when the exchange field gets very large. This is seen in Fig. 3(c), for the $\left(d_{x^{2}-y^{2}}+i s\right)$-wave pairing state, where as the exchange field $x$ increases the conductance peaks are reduced symmetrically. In the $d_{x^{2}-y^{2}}+i d_{x y}$ a dip develops within the subgap region as seen in Fig $4(\mathrm{c})$. The $E=0$ value is more sensitive to the exchange field (i.e., the Andreev reflection coefficient goes to zero faster) and the tunneling conductance for $E=0$, is suppressed more easily as the exchange field increases. In both pairing states the reduction of the subgap conductance is symmetric since the density of states modulation within the subgap is not induced by spin-dependent effects, for example, a magnetic field. In that case we would expect an asymmetric evolution with the exchange field $x$ since the effect of the magnetic field depends on the spin of the incident quasiparticle. This has been obtained in Ref. [10] where the tunneling conductance in a ferromagnet/insulator $/\left(d_{x^{2}-y^{2}}+i s\right)$-wave superconductor and also the effect of the magnetic field $H$ in a ferromagnet/insulator $/\left(d_{x^{2}-y^{2}}\right)$-wave superconductor, is studied in order to identify the mechanism responsible for the splitting of the zero energy conductance peak in high- $T_{c}$ superconductors. In the $\left(d_{x^{2}-y^{2}}+i s\right)$-wave state for $z_{0}=2.5, \beta=0$, and $x=0$, 
as seen in Fig. 3(b) there are no states within the subgap and $\sigma_{q}(E)$ reduces to zero there. In the $d_{x^{2}-y^{2}}+i d_{x y}$ for $z_{0}=2.5, \beta=0$ as seen in Fig. (b) there are residual values within the subgap that are suppressed as $x$ gets larger. For $z_{0}=0$ the evolution of the pairing state with the exchange field is similar in the two pairing states as seen in Figs. B(a), E(a).

\section{SUPPRESSION OF THE BOUND STATE ENERGIES}

We examine the evolution of the bound states with the exchange field $x$. The equation giving the energy peak level is written as

$$
\left.\phi_{-} \phi_{+}^{*} n_{+} n_{-}\right|_{E=E_{p}}=1.0 .
$$

When this condition occurs the Andreev- and normal-reflection amplitudes $a_{\uparrow[\downarrow]}, b_{\uparrow[\downarrow]}$ for the spin up(down) quasiparticles are reduced to

$$
\begin{gathered}
a_{\uparrow[\downarrow]}=\frac{2 n_{+} \lambda_{1}}{\lambda_{1}+\lambda_{2}}, \\
b_{\uparrow[\downarrow]}=\frac{\lambda_{1}-\lambda_{2}}{\lambda_{1}+\lambda_{2}} .
\end{gathered}
$$

In Fig. 5(a) we plot the magnitude of the Andreev reflection amplitude for spin-up(-down) quasiparticle $\operatorname{Re} \frac{\lambda_{2}}{\lambda_{1}}\left|a_{\uparrow[\downarrow]}\right|^{2}$ as a function of the exchange field $x$ for $\beta=\pi / 4, z_{0}=2.5$, for the $\left(d_{x^{2}-y^{2}}+i s\right)$-wave case. The corresponding magnitude of the normal-reflection amplitude is plotted in Fig. 5bl. The energy is equal to the amplitude of the $s$-wave component ( $\left.E=0.3 \Delta_{0}\right)$ for which bound states are formed for $0<\theta<\pi / 2$, when the ferromagnet is normal metal (i.e. $x=0$ ). For $\theta=\pi / 4, \pi / 8$, and $x=0$, where $\lambda_{1}=\lambda_{2}$, the Andreev reflection coefficient is equal to 1 , and the normal-reflection coefficient is equal to 0 , as obtained from Eqs. 18 and 19. In this case the conductance peak is due to the normal-state conductance in Eq. 14 that varies as $1 / z_{0}^{2}$. As $x$ increases the amplitude of the Andreevreflection decays to zero at a critical value $x_{c}$ that depends from the angle $\theta$ for which bound state occurs. The amplitude of the normal reflection increases with the exchange 
field. The suppression of the Andreev reflection amplitude with $x$, explains the reduction of the conductance peaks as $x$ increases. In the space of $\theta, x$ the critical exchange field $x_{c}$ is defined from the separating line between the VAR region and the AR region in Fig. 2. For trajectories $\theta$ that correspond to bound states the Andreev reflection vanishes within the VAR region. The critical exchange field $x_{c}$ is maximum $\left(x_{c}=1\right)$ when the bound state is at $\theta=0$ and is reduced to zero as $\theta$ moves toward $\theta= \pm \pi / 2$. This is also seen in Figs. 5(a) and 5(b) where for $\theta=\pi / 8, x_{c}=0.75$, while for $\theta=\pi / 4, x_{c}=0.33$. For a given value of the exchange field $x$, a bound state at $\theta$ contributes to the $\sigma(E)$ only if $x<x_{c}(\theta)$. This means that as $x$ increases the range of bound states that contributes to the tunneling conductance, is reduced and the peaks are suppressed.

In the $\left(d_{x^{2}-y^{2}}+i d_{x y}\right)$-wave case when the ferromagnet is a normal metal, $(x=0)$ the bound states occur for discrete values of the quasiparticle angle $\theta$, for fixed $\beta$. The Andreev reflection coefficient is equal to 1 for these values of $\theta$. When the exchange field increases the Andreev (normal-)-reflection coefficient goes to $0(1)$ at a critical value $x_{c}$. This is seen in Fig. 6(a) and 6(b) for two different pairs of $(E, \theta)$ for which bound state is formed for $x=0$, i.e., $(E=0, \theta=\pi / 4)$ and $\left(E=0.3 \Delta_{0}, \theta=0\right)$ [8]. The critical value of $x$ for which the Andreev reflection coefficient goes to zero is independent from the pairing potential, and also from the energy of the bound state. It depends only on the angle $\theta$. This is seen in Figs. 5 and 6, where for $\theta=\pi / 4$ the critical exchange field is $x_{c}=0.33$ for both pairing states and for different values of the bound-state energy, i.e., $E=0.3 \Delta_{0}$ and $E=0$ correspondingly. The variation of the bound state angle $\theta$ with $x_{c}$ seen in Fig. 2 holds also for bound states in the $\left(d_{x^{2}-y^{2}}+i d_{x y}\right)$-wave state, and can be used to explain the suppression of the tunneling conductance at zero energy seen in Fig. $1(\mathrm{c})$ with the exchange field $x$ as follows. For $E=0.3 \Delta_{0}$ a bound state exists at $\theta=0$ [8] for which $x_{c} \approx 1$ is maximum. For $x<x_{c}$ the incident electrons are Andreev-reflected and the tunneling conductance has a finite value. The variation of the Andreev-reflection amplitude with $x$ is seen in Fig. 6a. For the same energy another bound state exists at $\theta=\pi / 2$, which does not contribute to the Andreev reflection since $x_{c}$ is zero for this bound state. However, for $E=0$ one bound state is 
formed for values of $\theta$ close to $-\pi / 2$ where $x_{c}$ is close to zero and the Andreev reflection does not occurs. The other bound state at $E=0$ occurs for $\theta=\pi / 4$ and contributes to the Andreev reflection up to $x=0.33$, as seen in Fig. 6(a). For $x>0.33$ the Andreev-reflection amplitude is zero and also the tunneling conductance is suppressed. This is seen in Fig. ๒(c) for $E=0$ and $x=0.4$ (dotted line). Therefore the tunneling conductance at $E=0$ decays to zero more rapidly with $x$ than the conductance at $E=0.3 \Delta_{0}$. For a combination of $(E, \theta)$ for which no bound state is formed, the Andreev reflection amplitude is suppressed for all values of the exchange interaction $x$ indicating that the exchange field mainly affects the bound states.

\section{MAGNETIC FIELD EFFECTS}

In this section we describe the effect of the external magnetic field $H$ in the spectra for different values of the exchange field $x$. We will see that since the effect of the magnetic field depends on the spin, the evolution of the tunneling spectra with $x$ is asymmetric. The tunneling conductance is given by

$$
\sigma_{q}(E)=\sigma_{q_{\uparrow}}\left(E-\mu_{B} H\right)+\sigma_{q_{\downarrow}}\left(E+\mu_{B} H\right) .
$$

In Fig. $7(\mathrm{a})$ and $\mathbb{7}(\mathrm{b})$ the tunneling conductance $\sigma_{q}(E)$ is plotted for fixed magnetic field $\mu_{B} H / \Delta_{0}=0.2$, and barrier strength $z_{0}=2.5$, for different values of the exchange interaction $x$. The pairing symmetry of the superconductor is $d_{x^{2}-y^{2}}+i s$ and $d_{x^{2}-y^{2}}+i d_{x y}$, respectively. The orientation of the superconductor is chosen as $\beta=\pi / 4$.

In the absence of the exchange interaction $(x=0)$ the magnetic field splits symmetrically the tunneling spectrum that is a linear superposition of the spectra for spin up(down) quasiparticles. The amplitude of the spliting depends linearly on the magnetic field $H$. For the case of $\mu_{B} H / \Delta_{0}=0.2$, seen in Fig. 7 the spin-up(-down) part of the spectra partially overlap while for larger values of the magnetic field the spin-up and -down branches are well separated. In the latter case the left(right) branch of the spectra originates from spin-up(down) quasiparticle spectra $\sigma_{q_{\uparrow}}\left(E-\mu_{B} H\right)\left[\sigma_{q_{\downarrow}}\left(E+\mu_{B} H\right)\right]$. 
For the $d_{x^{2}-y^{2}}+i s$-wave case the condition for the formation of bound states is slightly modified under the presence of magnetic field to $\left|E-\mu_{B} H\right|=\Delta_{2}$, for the spin-up region, and $\left|E+\mu_{B} H\right|=\Delta_{2}$, for the spin-down, from the corresponding $|E|=\Delta_{2}$ in the absence of any field. So the multiplication of the $\left(d_{x^{2}-y^{2}}+i s\right)$-wave pairing state and the presence of magnetic field results into the appearance of four peaks in the conductance spectra, which in the limit of $x=0$ have equal heights.

The main effect of the polarization is the imbalance in the peak heights for $E$ positive and negative. The ratio of the peaks for positive and negative energy is proportional to the exchange field of the material. This can be extracted from the different evolution of the Andreev- and normal-reflection coefficients for spin-up and -down quasiparticles with the exchange field seen in Figs. . modified in the presence of the magnetic field, the analysis concerning the above Figures still holds for the modified energies. For a given energy and angle $\theta$ for which bound state occurs the quantities $\bar{\sigma}_{q_{\uparrow}}(E, \theta), \bar{\sigma}_{q_{\downarrow}}(E, \theta)$, have different values causing the asymmetricity in the peak heights for the spin-up and -down part of the spectrum. The asymmetricity in the Andreev reflection coefficient can also be seen in Fig. 8a, for spin up(down) quasiparticles as solid (dotted) line, as a function of the energy $E / \Delta_{0}$ for fixed exchange field $x=0.6$, and the pairing symmetry of the superconductor is $d_{x^{2}-y^{2}}+i s$. The same characteristic appears in the normal reflection coefficient that is plotted in Fig. 8(b). The peaks in the Andreev-reflection coefficient are formed at the bound state energies and due to the finite exchange interaction are suppressed from the unit. The same result is plotted in Fig. 9(a) and 9 (b), for the $\left(d_{x^{2}-y^{2}}+i d_{x y}\right)$-wave pairing symmetry. Here the bound state is formed for a particular value of $\theta=0$. Other reasons for the asymmetricity of the spectra for the spin up(down) quasiparticle are the factors $P_{\uparrow[\downarrow]}$ and $q_{\uparrow[\downarrow]}$ that appear in the definition of the tunneling conductance Eq. (12). 


\section{C-AXIS TUNNELING}

In the preceding sections we discussed the tunneling effect in two dimensional models. In this section we discuss the tunneling effect along the $c$ axis that takes into account threedimensional effects. A semi-infinite double layer structure is assumed and the volume of the integration is taken as the three dimensional half sphere. The interface is perpendicular to the $z$ axis and is located at $z=0$ as seen in Fig. 10. Suppose that an electron is injected from the ferromagnet with polar angle $\theta$ and azimuthal angle $\phi$. The electron like (hole) like quasiparticle will experience different pair potentials $\Delta_{\rho \rho^{\prime}}\left(\theta_{+}\right)\left[\Delta_{\rho \rho^{\prime}}\left(\theta_{-}\right)\right]$, where $\theta_{+}=\theta$ and $\theta_{-}=\pi-\theta$, and the quantities $\rho, \rho^{\prime}$ denote spin indices. The coefficients of the Andreev and normal reflection are obtained by solving the BdG equations under the following boundary conditions

$$
\begin{gathered}
\left.\Psi(\mathbf{r})\right|_{z=0_{-}}=\left.\Psi(\mathbf{r})\right|_{z=0_{+}} \\
\left.\frac{d \Psi(\mathbf{r})}{d z}\right|_{z=0_{-}}=\left.\frac{d \Psi(\mathbf{r})}{d z}\right|_{z=0_{+}}-\left.\frac{2 m V}{\hbar^{2}} \Psi(\mathbf{r})\right|_{z=0_{-}}
\end{gathered}
$$

Using the obtained coefficients the tunneling conductance is calculated using the formula given in the preceding sections,

$$
\sigma_{q_{\uparrow[\downarrow]}}(E)=\frac{1}{R_{N}} \int_{0}^{\pi / 2} \int_{0}^{2 \pi} \cos \theta \sin \theta \bar{\sigma}_{q_{\uparrow[\downarrow]}}(E, \theta, \phi) P_{\uparrow[\downarrow]} q_{\uparrow[\downarrow]} d \theta d \phi
$$

where

$$
\begin{gathered}
R_{N}=\int_{0}^{\pi / 2} \sin \theta \cos \theta\left[\sigma_{N_{\uparrow}}(\theta) P_{\uparrow} q_{\uparrow}+\sigma_{N_{\downarrow}}(\theta) P_{\downarrow} q_{\downarrow}\right] d \theta d \phi, \\
\sigma_{N_{\uparrow[\downarrow]}}(\theta)=\frac{4 \lambda_{1}}{\left(1+\lambda_{1}\right)^{2}+z_{\uparrow[\downarrow]}^{2}} .
\end{gathered}
$$

The pairing potentials are given by

a) In the $\left(d_{x^{2}-y^{2}}+i s\right)$-wave case

$$
\Delta(\theta, \phi)=\Delta_{1} \cos 2 \phi+i \Delta_{2},
$$


b) In the $\left(d_{x^{2}-y^{2}}+i d_{x y}\right)$-wave case

$$
\Delta(\theta, \phi)=\Delta_{1} \cos 2 \phi+i \Delta_{2} \sin 2 \phi .
$$

Figures 11(a) and 11(b) show the calculated conductance spectra for various exchange potentials. Unlike the case where the interface is perpendicular to the $x$ axis the tunneling spectra is similar for the $d_{x^{2}-y^{2}}+i s, d_{x^{2}-y^{2}}+i d_{x y}$ cases. Also a subgap region is formed within the energy gap due to the nodeless form of the order parameter. The conductance peaks are absent since the transmitted quasiparticles do not feel a sign change of the pair potential on the Fermi surface. The case of $c$-axis tunneling has been treated experimentally using scanning tunneling microscopy or point-contact spectroscopy, for the case of normal metal / superconductor junction [17,18]. No zero energy peak and a clear $V$-like line shape for the $c$-axis tunneling conductance has been observed. The calculated spectra for the $d_{x^{2}-y^{2}}+i s$, $d_{x^{2}-y^{2}}+i d_{x y}$ states is flattened out for $E<\Delta_{2}$ that contradicts the experimental data. The $\left(d_{x^{2}-y^{2}}\right)$-wave order parameter does not change sign and is not suppressed at the $c$-axis surface. So the attractive interaction in the subdominant pairing channel is small relative to the dominant and a transition to a state breaking $\mathcal{T}$ does not happen. On the other hand a mixed order parameter such as the $d_{x^{2}-y^{2}}+s$ may exists due to the orthorhombic distortion of the lattice, as seen in the $c$-axis Josephson experiments.

\section{CONCLUSIONS}

We calculated the tunneling conductance in ferromagnet / insulator / superconductor, junction using the BTK formalism. We assumed two possible pairing potentials for the superconductor that break the time-reversal symmetry, i.e., $d_{x^{2}-y^{2}}+i s, d_{x^{2}-y^{2}}+i d_{x y}$. The evolution of the spectra with the exchange field is the same for $z_{0}=0$ but different in the tunneling limit where $z_{0}$ is large, and can be considered as a probe for time reversal symmetry broken pairing states. The weak Andreev reflection within the ferromagnet results

in the suppression of the tunneling conductance and eliminates the resonances due to the 
anisotropy of the pair potential. The evolution of the tunneling conductance within the gap is symmetric since the splitting is not induced from spin-dependent effects, for example, a magnetic field, but from the nodeless form of the pairing potential.

We also derived that the condition for a bound state at angle $\theta$ to contribute to the Andreev-reflection and hence to the tunneling conductance, for a given value of the exchange interaction $x$ is $x<x_{c} . x_{c}$ is the critical exchange field for which the Andreev refletion coefficient goes to zero and is given from the separating line between the VAR region and the AR region in the space of $\theta, x$. This condition was used to explain the suppression of the conductance around $E=0$ with the exchange field in the $\left(d_{x^{2}-y^{2}}+i d_{x y}\right)$-wave state.

The magnetic field splits linearly the tunneling spectra, and the exchange potential induces an imbalance in the peak heights for positive and negative energies. The asymmetricity in the peak heights originates from the different evolution of the Andreev- and normal-reflection amplitudes at the bound state energies with the exchange field.

The $c$-axis tunneling from ferromagnet to superconductor does not show any differences between $\mathcal{T}$-broken pairing states since the transmitted quasiparticles experience the same sign of the pairing potential.

Throughout this paper the order parameter is not calculated self-consistently. However, since the characteristics of the tunneling conductance depend mainly from the angular part of the pairing potential, the essential results are expected to change only quantitatively when the suppression of the order parameter near the surface is taken into account. 


\section{REFERENCES}

[1] M. Matsumoto and H. Shiba, J. Phys. Soc. Jpn. 64, 3384 (1995)

[2] G.E. Blonder, M. Tinkham, and T.M. Klapwijk, Phys. Rev. B 25, 4515 (1982).

[3] A.F. Andreev, Zh. Eksp. Teor. Fiz. 46, 1823 (1964) [Soviet Phys. JETP, 19, 1228 (1964)].

[4] C.R. Hu, Phys. Rev. Lett. 72, 1526 (1994).

[5] Y. Tanaka, and S. Kashiwaya, Phys. Rev. Lett. 74, 3451 (1995).

[6] M. Covington, M. Aprili, E. Paraoanu, L.H. Green, F. Xu, J. Zhu, and C.A. Mirkin, Phys. Rev. Lett. 79, 277 (1997).

[7] S. Kashiwaya, Y. Tanaka, M. Koyanagi, and K. Kajimura, Phys. Rev. B 53, 2667 (1996).

[8] N. Stefanakis, J. Phys.: Condens. Matter 13, 1265 (2001).

[9] M.J.M. de Jong and C.W.J. Beenakker, Phys. Rev. Lett. 74, 1657 (1995).

[10] S. Kashiwaya, Y. Tanaka, N. Yoshida and M.R. Beasley, Phys. Rev. B 60, 3572 (1999).

[11] J.-X. Zhu, B. Friedman and C.S. Ting, Phys. Rev. B 59, 9558 (1999).

[12] I. Zutic and O.T. Valls, Phys. Rev. B 61, 1555 (2000).

[13] N. Stefanakis, J. Phys.: Condens. Matter 13, 3643 (2001).

[14] Z. Rodovic, L. Dobrosavljevic-Grujic, A.I. Buzdin, and J.R. Clem, Phys. Rev. B 38, 2388 (1988).

[15] Cr. Bruder, Phys. Rev. B 41, 4017 (1990).

[16] Y. Tanaka, Y. Tanuma, and S. Kashiwaya, cond-mat/0101277, unpublished.

[17] J.Y.T. Wei, N.-C. Yeh, D.F. Garrigus, and M. Strasik, Phys. Rev. Lett. 81, 2542 (1998). 
[18] S. Kashiwaya, Y. Tanaka, M. Koyanagi, H. Takashima, and K. Kajimura, Phys. Rev. B 51, 1350 (1995). 


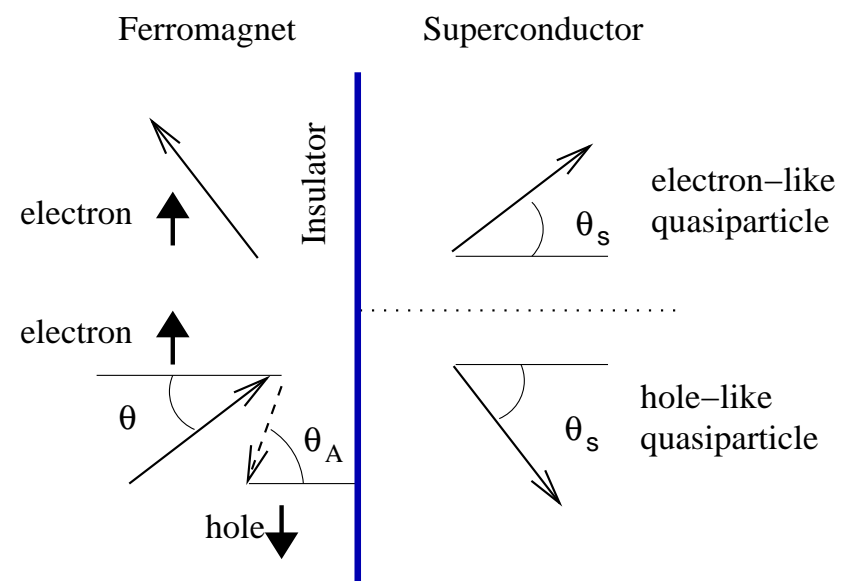

FIG. 1. The geometry of the ferromagnet / insulator / singlet superconductor interface. The vertical line along the $y$ axis represents the insulator. The arrows illustrate the transmission and reflection processes at the interface. $\theta$ is the angle of the incident electron and the normal, $\theta_{A}$ is the angle of the reflected-hole trajectory and the normal, and $\theta_{s}$ is the angle of the transmitted quasiparticle and the normal. Note that $\theta$ is not equal to $\theta_{A}$ since the retroreflection of the Andreev process is lost. In the Andreev-reflection process an electron with spin up is Andreev reflected as a hole with spin down and normally reflected as an electron with spin-up.

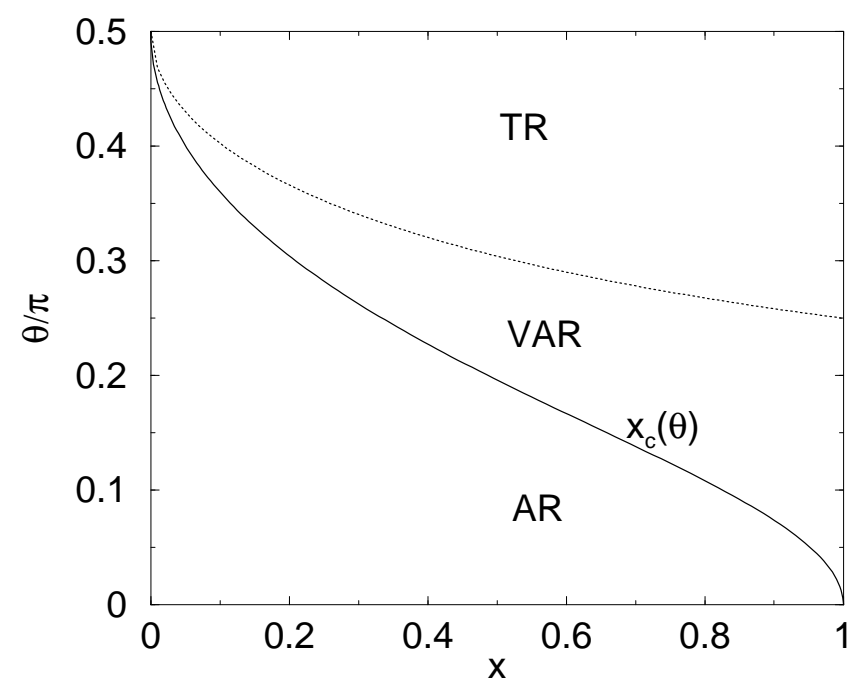

FIG. 2. The regions of the space of $\theta, x$ TR, where total reflection occurs, VAR where Andreev reflected quasiparticles do not propagate, while the transmitted quasiparticles propagate, AR where Andreev reflection occurs. 

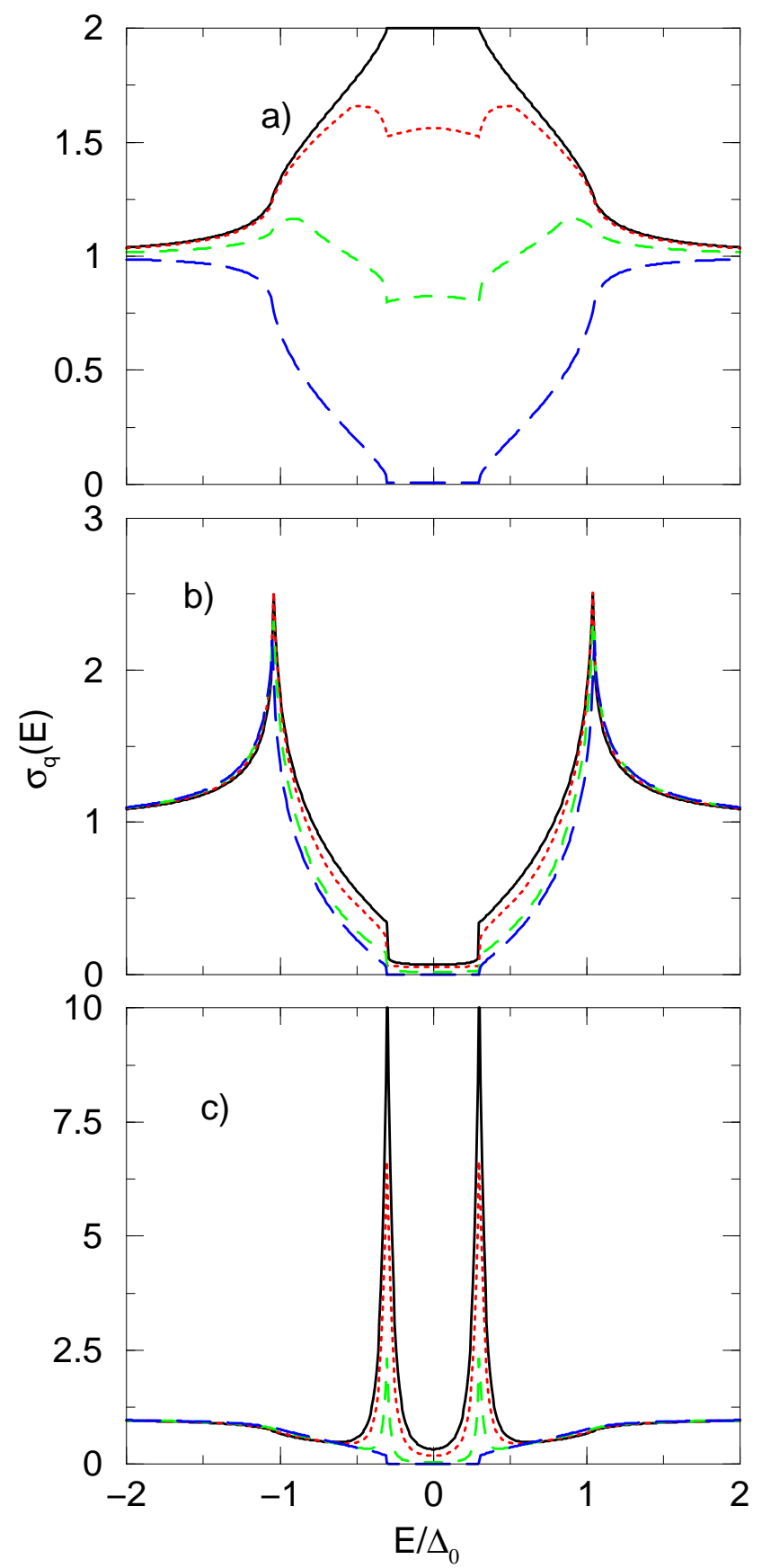

FIG. 3. Normalized tunneling conductance $\sigma_{q}(E)$ as a function of $E / \Delta_{0}$ for $x=0$ (solid line), $x=0.4$ (dotted line), $x=0.8$ (dashed line), and $x=0.999$ (long-dashed line), for different orientations (a) $z_{0}=0, \beta=0,(\mathrm{~b}) z_{0}=2.5, \beta=0$, (c) $z_{0}=2.5, \beta=\pi / 4$. The pairing symmetry of the superconductor is $d_{x^{2}-y^{2}}+i s$ with $\Delta_{1}=\Delta_{0}, \Delta_{2}=0.3 \Delta_{0}$. 

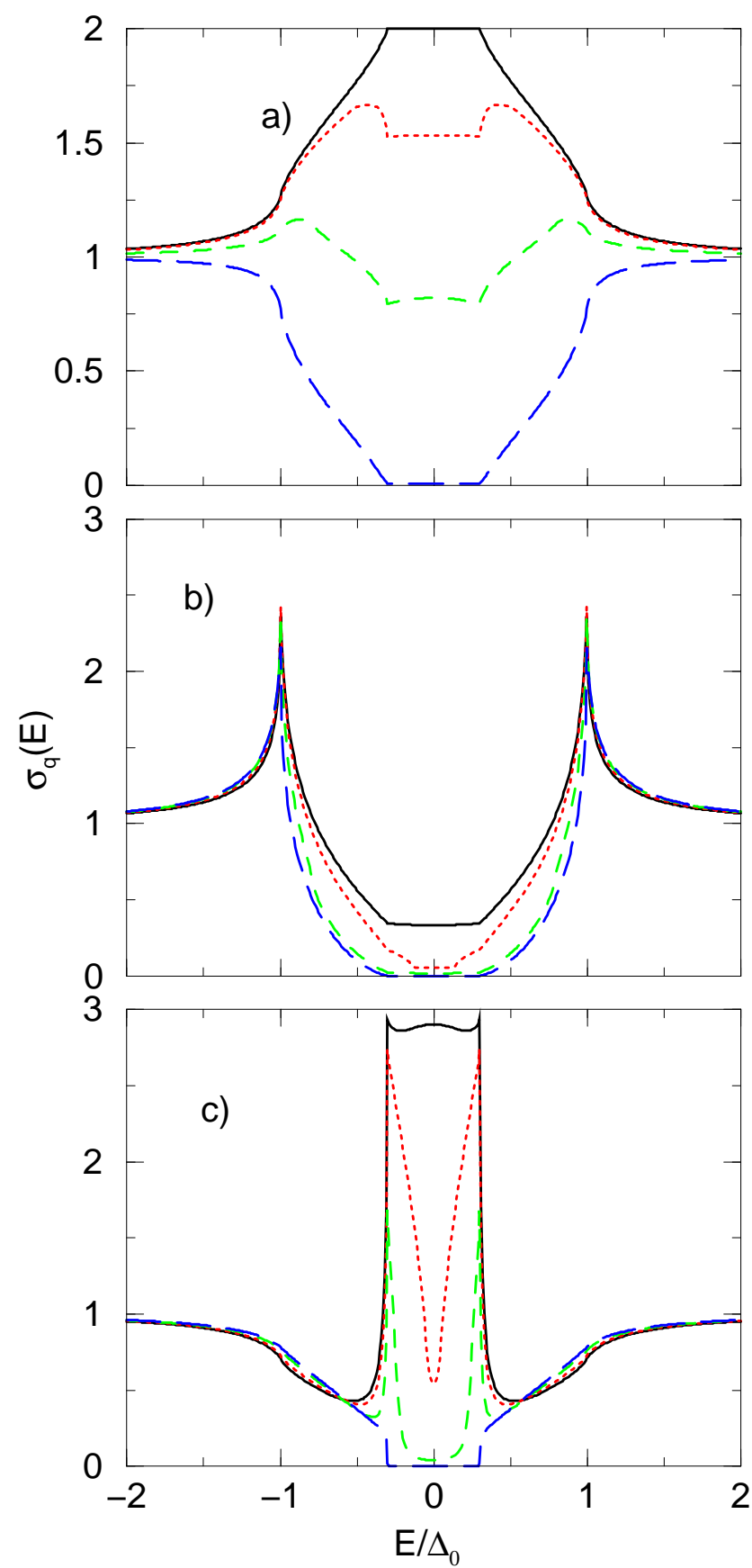

FIG. 4. The same as in Fig. 2. The pairing symmetry of the superconductor is $d_{x^{2}-y^{2}}+i d_{x y}$, with $\Delta_{1}=\Delta_{0}, \Delta_{2}=0.3 \Delta_{0}$ 

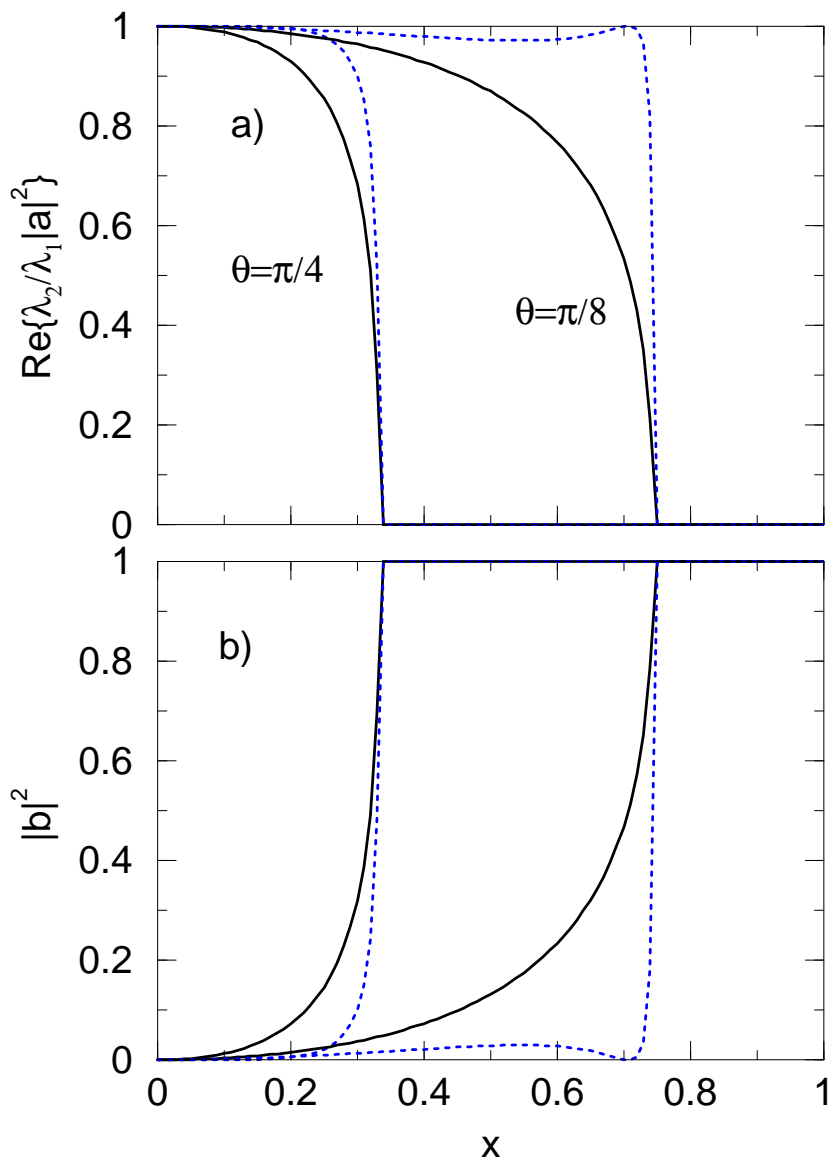

FIG. 5. a) The magnitude of the Andreev reflection coefficient $\operatorname{Re} \frac{\lambda_{2}}{\lambda_{1}}\left|a_{\uparrow[\downarrow]}\right|^{2}$ as a function of the exchange field $x$, for spin-up(-down) quasiparticles, solid (dotted) line, for $\theta=\pi / 4$ and $\theta=\pi / 8$, at the bound-state energy $E=0.3 \Delta_{0}$. The pairing symmetry of the superconductor is $d_{x^{2}-y^{2}}+i s$ and $\beta=\pi / 4$. b) The corresponding magnitude of the normal-reflection coefficient $\left|b_{\uparrow[\downarrow]}\right|^{2}$ as a function of the exchange field $x$. 

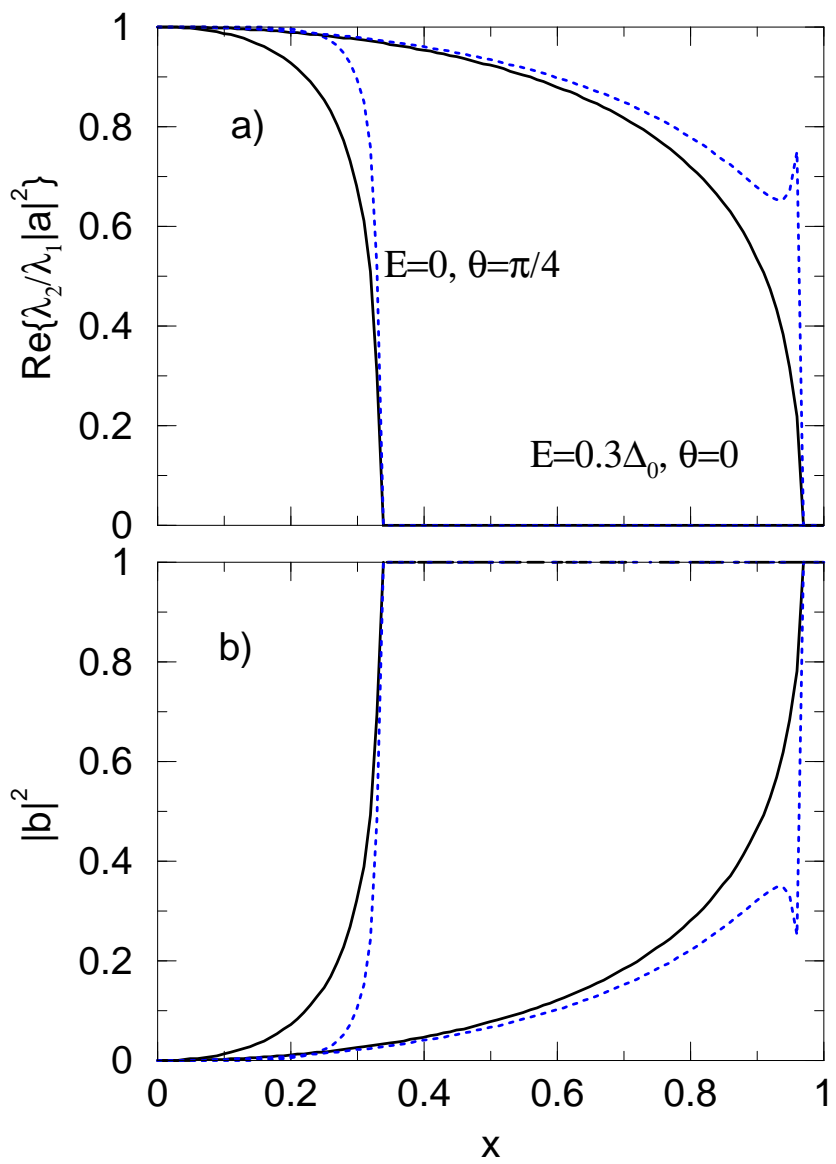

FIG. 6. The magnitude of the Andreev reflection coefficient $\operatorname{Re} \frac{\lambda_{2}}{\lambda_{1}}\left|a_{\uparrow[\downarrow]}\right|^{2}$ (a) and normal-reflection coefficient $\left|b_{\uparrow[\downarrow]}\right|^{2}$ (b) as a function of the exchange field $x$, for spin-up(-down) quasiparticles, solid (dotted) line, for two pairs of $(E, \theta)$, for which bound states occur i.e. $(E=0, \theta=\pi / 4)$, and $\left(E=0.3 \Delta_{0}, \theta=0\right)$ in a superconductor with $\left(d_{x^{2}-y^{2}}+i d_{x y}\right)$-wave pairing and $\beta=\pi / 4$. 

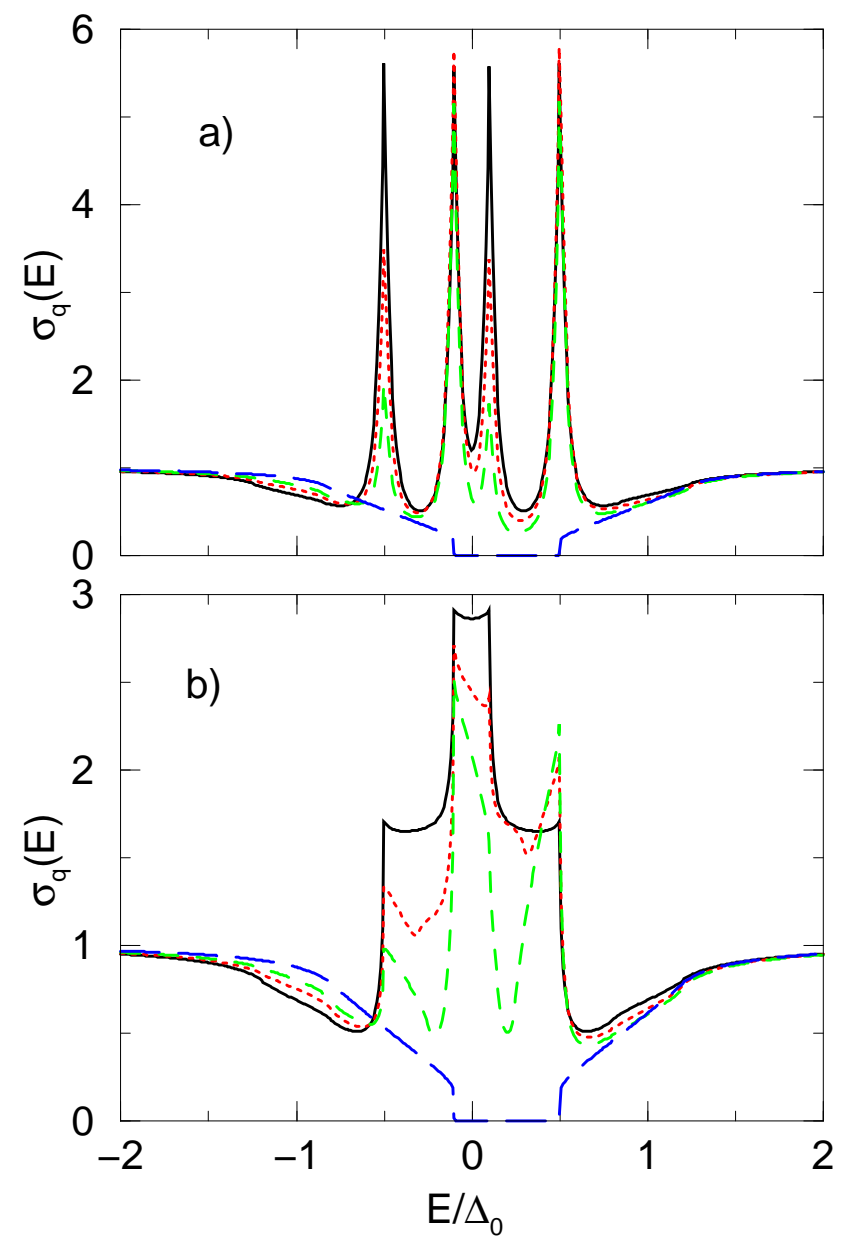

FIG. 7. Normalized tunneling conductance $\sigma_{q}(E)$ as a function of $E / \Delta_{0}$ for $x=0$ (solid line), $x=0.2$ (dotted line), $x=0.4$ (dashed line), and $x=0.999$ (long-dashed line), for $z_{0}=2.5$, $\beta=\pi / 4$, in the presence of an external magnetic field $\mu_{B} H / \Delta_{0}=0.2$. The pairing symmetry of the superconductor is (a) $d_{x^{2}-y^{2}}+i s$ with $\Delta_{1}=\Delta_{0}$ and $\Delta_{2}=0.3 \Delta_{0}$. (b) $d_{x^{2}-y^{2}}+i d_{x y}$ with $\Delta_{1}=\Delta_{0}$ and $\Delta_{2}=0.3 \Delta_{0}$. 

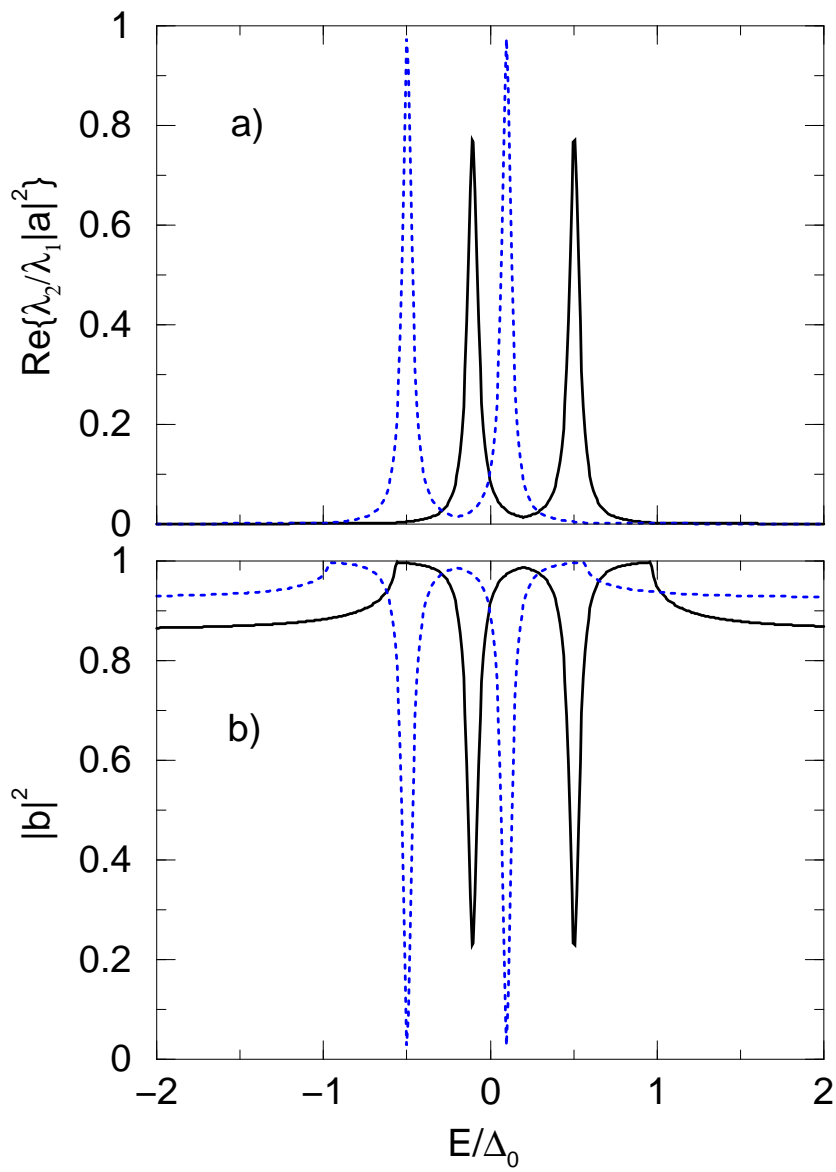

FIG. 8. a) The magnitude of the Andreev reflection coefficient $\operatorname{Re} \frac{\lambda_{2}}{\lambda_{1}}\left|a_{\uparrow[\downarrow]}\right|^{2}$ as a function of $E / \Delta_{0}$, for spin-up(-down) quasiparticles, solid (dotted) line, for $\theta=\pi / 8$, for exchange field $x=0.6$, $\mu_{B} H / \Delta_{0}=0.2$. The pairing symmetry of the superconductor is $d_{x^{2}-y^{2}}+i s$ and $\beta=\pi / 4$. b) The corresponding magnitude of the normal-reflection coefficient $\left|b_{\uparrow[\downarrow]}\right|^{2}$ as a function of $E / \Delta_{0}$. 

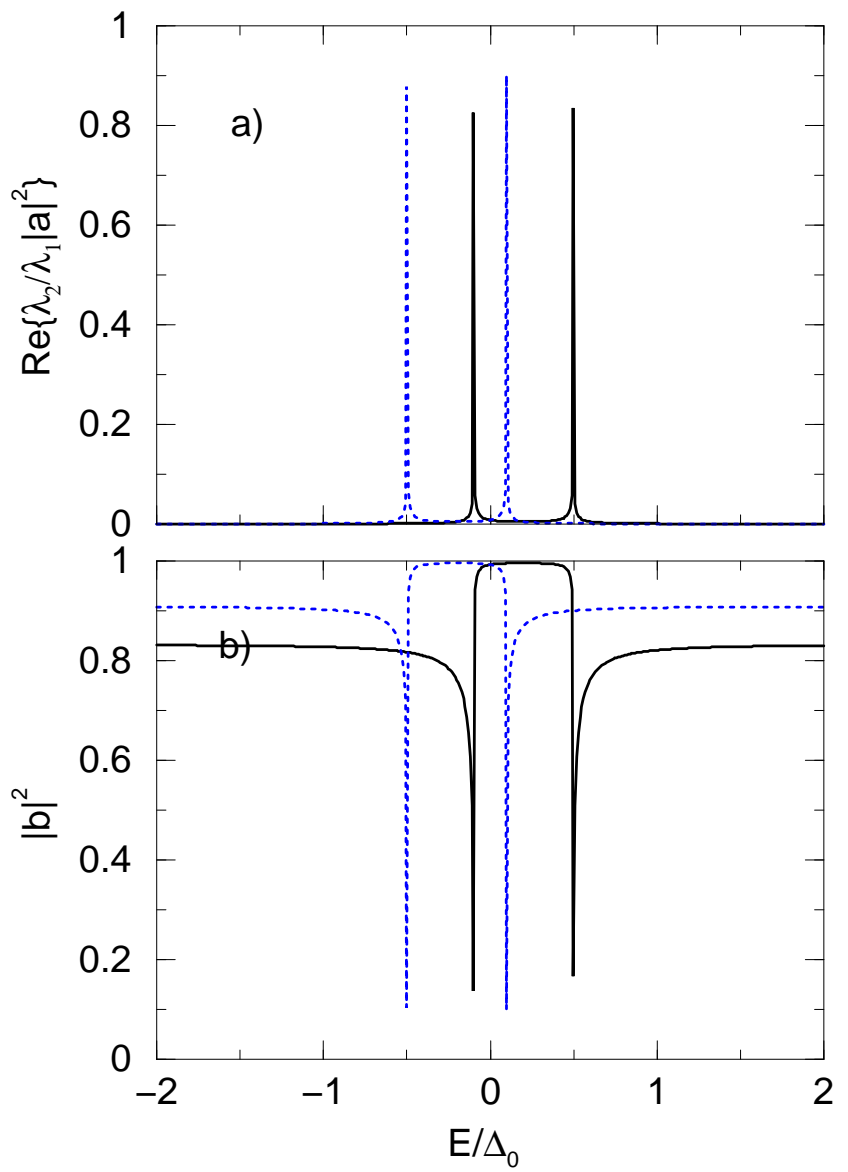

FIG. 9. a) The magnitude of the Andreev reflection coefficient $\operatorname{Re} \frac{\lambda_{2}}{\lambda_{1}}\left|a_{\uparrow[\downarrow]}\right|^{2}$ as a function of $E / \Delta_{0}$, for spin-up(-down) quasiparticles, solid (dotted) line, for $\theta=0$, for exchange field $x=0.6$, $\mu_{B} H / \Delta_{0}=0.2$. The pairing symmetry of the superconductor is $d_{x^{2}-y^{2}}+i d_{x y}$, and $\beta=\pi / 4$. b) The corresponding magnitude of the normal-reflection coefficient $\left|b_{\uparrow[\downarrow]}\right|^{2}$ as a function of $E / \Delta_{0}$. 


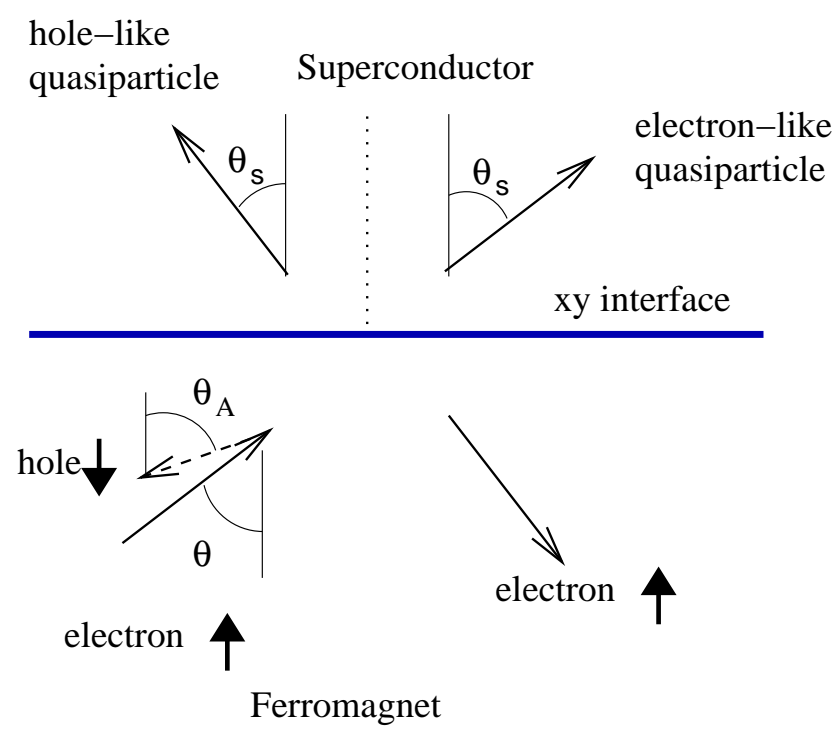

FIG. 10. The figure illustrate the transmission and reflection processes of the quasiparticle at the interface of the junction with $x y$ plane interface. 

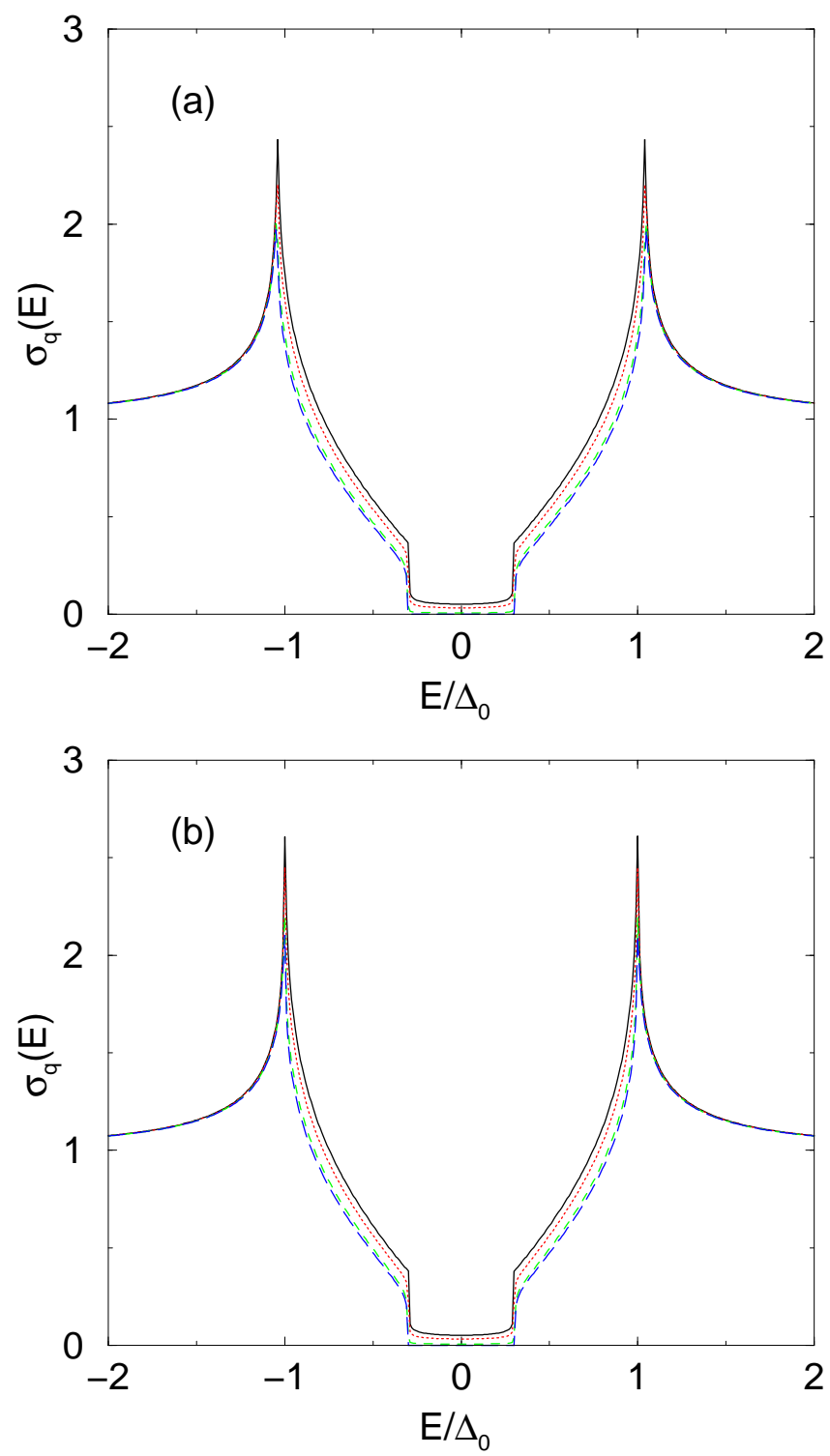

FIG. 11. Normalized tunneling conductance $\sigma_{q}(E)$ as a function of $E / \Delta_{0}$ for $x=0$ (solid line), $x=0.4$ (dotted line), $x=0.8$ (dashed line), and $x=0.999$ (long dashed line), for $z_{0}=2.5$. The interface is perpendicular to the $z$ axis. The pairing symmetry of the superconductor is (a) $d_{x^{2}-y^{2}}+i s$ with $\Delta_{1}=\Delta_{0}$ and $\Delta_{2}=0.3 \Delta_{0}$. (b) $d_{x^{2}-y^{2}}+i d_{x y}$ with $\Delta_{1}=\Delta_{0}$ and $\Delta_{2}=0.3 \Delta_{0}$. 